\title{
High-spatial-resolution X-ray Imaging by Scintillator in Silicon Collimator
}

\author{
Kento Tabata, ${ }^{1}$ Ryota Ohtake, ${ }^{2}$ and Toru Aoki ${ }^{1,2,3^{*}}$ \\ ${ }^{1}$ Graduate School of Science and Technology, Shizuoka University, \\ 3-5-1, Johoku, Hamamatsu, Shizuoka 432-8011, Japan \\ ${ }^{2}$ Graduate School of Integrated Science and Technology, Shizuoka University, \\ 3-5-1, Johoku, Hamamatsu, Shizuoka 432-8011, Japan \\ ${ }^{3}$ Research Institute of Electronics, Shizuoka University, 3-5-1, Johoku, Hamamatsu, Shizuoka 432-8011, Japan
}

(Received June 18, 2020; accepted November 9, 2020)

Keywords: X-ray detector, high-spatial-resolution scintillator detector, thallium-doped cesium iodide, collimator, modulation transfer function, thallium concentration, optical separation, pixel structures

The purpose of this paper is to improve the spatial resolution of scintillator X-ray image detectors. The spatial resolution of these detectors is lower than that of other direct conversion detectors because of the diffusion of visible light in the scintillator. This problem was solved by using a collimator manufactured from a silicon wafer. The silicon wafer was processed to make many small square holes in a grid pattern by a MEMS technique for the optical separation of visible light in the scintillator upon X-ray irradiation. Moreover, the structures of these grid holes were integrated with a silicon photodiode array to operate as a single detector. The properties of thallium-doped cesium iodide (CsI:Tl) depend on the $\mathrm{Tl}$ concentration. By optimizing the filling conditions of the scintillator for the silicon, collimator, the emission characteristics of CsI:Tl were adjusted to ensure their suitability for use with the Si photodiode, and the sealed state was observed by SEM and computed tomography (CT). CsI:Tl was encapsulated in a collimator under optimized conditions, and the improved spatial resolution was confirmed from the Modulation Transfer Function $(M T F)$. The pixel-structured scintillator with a silicon collimator was successfully fabricated, and our method shows the high-spatialresolution imaging performance of an indirect X-ray imaging detector.

\section{Introduction}

In the medical imaging and nondestructive inspection fields, there is increasing demand for larger detectors with high resolution. Scintillator-type imaging detectors have been widely used as indirect X-ray imaging detectors. These indirect detectors have advantages such as the fabrication of untiled large panels. Semiconductor detectors are tiled to detect large areas, but have areas that cannot be detected, such as junctions. The large scintillator-type detectors do not require tiling. The advantage of large-area panels is that the entire detector is within the detectable range. However, the spatial resolution of scintillator detectors is lower than that of

*Corresponding author: e-mail: aoki.toru@shizuoka.ac.jp

https://doi.org/10.18494/SAM.2020.2963 
other direct conversion X-ray imaging detectors owing to internal scattering and luminescence in the scintillator. ${ }^{(1,2)}$ Furthermore, the thicker the scintillator is for sensitivity, the worse the spatial resolution will be. ${ }^{(3)}$ Columnar crystal growth and a phase separation scintillator have been developed to suppress scattering and luminescence in the scintillator. ${ }^{(4-7)}$ Although the columnar structure significantly improves the trade-off between stopping power and spatial resolution, the light channeling is not perfect. ${ }^{(8)}$ In our study, we propose the processing of a silicon wafer to develop a scintillator with the higher spatial resolution of indirect X-ray imaging detectors. Large silicon wafers with a size of, for example, $450 \mathrm{~mm}$, are readily available, although they show poor spectral transmittance of visible light. In this study, the silicon wafer is processed to form a collimator by a microfabrication technique. The silicon collimator can achieve almost complete (99\%) optical separation of visible light upon X-ray irradiation of the scintillator, to solve the trade-off between stopping power and spatial resolution. In this paper, we demonstrate the improved spatial resolution of the scintillator-type X-ray imaging detector when we use it with a silicon collimator. CsI:Tl was used as the scintillator material because of its high luminosity and sensitivity to Si photodiodes. ${ }^{(9)}$ Our initial challenge was to find optimum conditions for filling the silicon collimator with the scintillator material as it is difficult to fill the scintillator owing to its high aspect ratio and the difference between the melting points of CsI and TII. The optimum conditions were adopted for the melting process used to fill the scintillator in the silicon collimator to achieve high packaging density. The luminescence characteristics of CsI:Tl depend on the Tl concentration, which is controlled by the heating duration in the melting process. Thereby, we achieved the optimum heating time for the melting process and obtained CsI:Tl with a $\mathrm{Tl}$ concentration that provides a suitable sensitivity in the wavelength range for the Si photodiode. Furthermore, we compared the performance of the X-ray scintillator with and without the silicon collimator in terms of spatial resolution.

\section{Materials and Methods}

\subsection{Silicon collimator}

Columnar crystal growth and phase separation have been adopted to improve the spatial resolution of scintillators, although the light channeling of the columnar structure is not perfect. In our study, a silicon wafer was processed to produce a silicon collimator with reduced scattering of light in the scintillator by forming small holes in a pixel pattern. The silicon collimator can achieve complete optical separation of visible light upon X-ray irradiation of the scintillator. $^{(10)}$ The silicon collimator contains the luminescence of the scintillator within the pixel cell and suppresses its diffusion by preventing optical crosstalk into adjacent pixels in the scintillator; the structure of these small holes can be integrated with a silicon photodiode array so that these structures can operate as a single detector unit. The fabrication procedure of the silicon collimator was as follows. The silicon collimator is made from a $400-\mu \mathrm{m}$-thick silicon wafer by a dry etching process. A 200 -nm-thick silicon oxide mask with a size of $90 \times 90 \mu \mathrm{m}^{2}$ was grown on the surface of the silicon wafer. The mask was perforated at a $100 \mu \mathrm{m}$ pitch using 
photoresist. Small square holes were drilled in the silicon wafer by photolithography using this mask. A cross-sectional schematic diagram of the silicon collimator is shown in Fig. 1(a) and a sectional view obtained from an optical microscope is shown in Fig. 1(b). This collimator can keep scintillated visible light optically separated. However, we were concerned about the effect of scattered X-rays on the materials on adjacent holes. Scattered X-ray radiation was simulated using a Monte Carlo particle and heavy ion transport code system (PHITS Ver. 2.88). ${ }^{(11)}$ A schematic diagram of this simulation is shown in Fig. 2(a). Pencil beam X-rays were applied to only one (incident pixel) of two CsI units separated by a silicon collimator. The numbers of generated photons absorbed in the incident pixel (A) and the adjacent pixel (B) were counted. The number of photons generated in CsI for each X-ray energy is shown in Fig. 2(b). As a result of integrating the number of absorbed photons, we obtained values of $2.0 \times 10^{7}$ for the incident

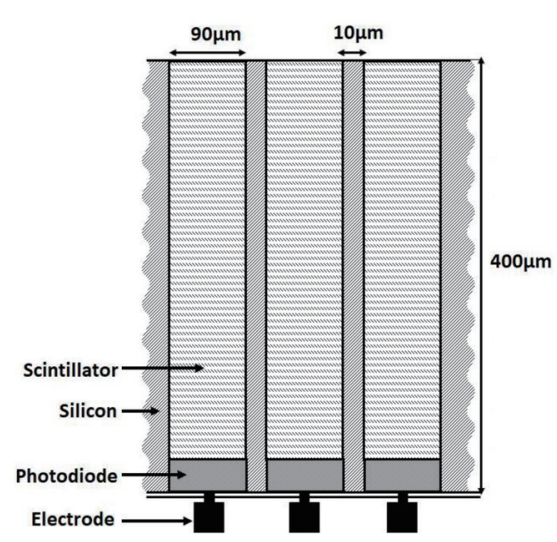

(a)

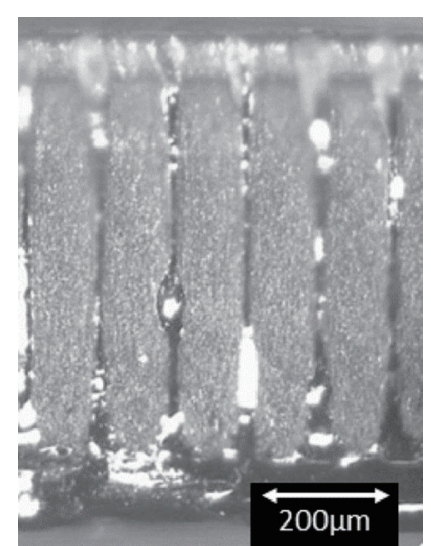

(b)

Fig. 1. Cross section of silicon collimator. (a) Schematic diagram. (b) Microscopic image.

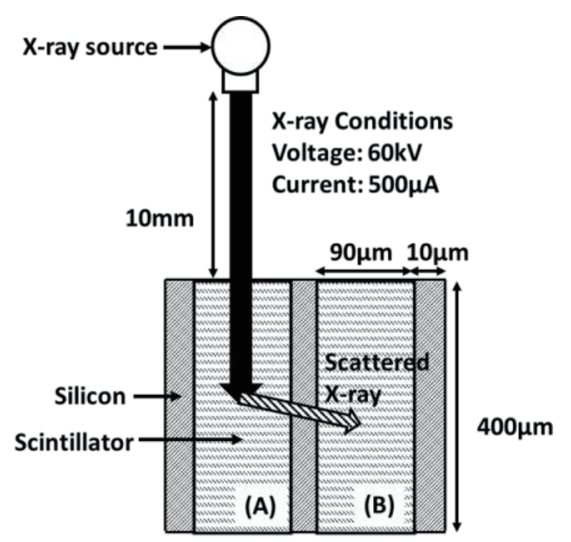

(a)

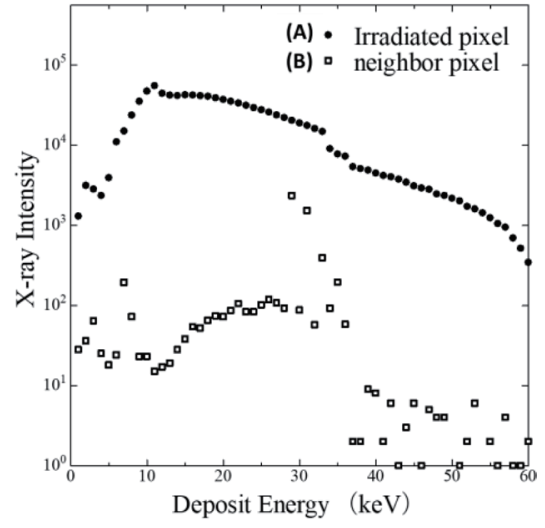

(b)

Fig. 2. (a) Schematic diagram of simulation of X-ray scattered radiation. (A) is a pixel directly irradiated by an $\mathrm{X}$-ray and (B) is a pixel adjacent to (A). (b) Result of the simulation showing the X-ray intensity of each energy band of (A) and (B). 
pixel and $1.8 \times 10^{5}$ for the adjacent pixel. From the results, we conclude that the influence of the scattered X-rays on the adjacent scintillators separated by the collimator is less than $1 \%$, which is considered to be small.

\subsection{Method of filling silicon collimator with CsI:TI}

CsI:Tl was encapsulated in a high-aspect-ratio silicon collimator by a melting process. A melted mixture of CsI and TII was poured onto the bottom of the collimator by gravity, and adhesion with the photodiode attached to the bottom was ensured. The melting points of CsI and TII are 894 and $713 \mathrm{~K}$, respectively. ${ }^{(12)}$ In the melting performed in the atmosphere, the evaporation rate of $\mathrm{Tl}$ is higher than that of CsI, and the ratio of CsI to $\mathrm{Tl}$ changes with the heating conditions. Since the characteristics of CsI:Tl depend on the Tl concentration, it is necessary to control the $\mathrm{Tl}$ concentration. Therefore, the temperature and concentration of $\mathrm{Tl}$ before heating were fixed at $973 \mathrm{~K}$ and $5 \mathrm{~mol} \%$, respectively. The concentration of $\mathrm{Tl}$ after heating was optimized by controlling the heating duration. The procedure for melting CsI:T1 was as follows. (1) The temperature of the resistance heating device was fixed at $973 \mathrm{~K}$. (2) The silicon substrate with CsI:Tl was placed on the heating device. (3) The silicon substrate was heated and CsI:Tl was melted onto the collimator holes. (4) After the assigned heating time had elapsed, the heating was stopped and the CsI:Tl-encapsulated collimator was naturally cooled to $293 \mathrm{~K}$ at a rate of $313 \mathrm{~K} / \mathrm{min}$.

Next, the filling state of CsI:Tl in the silicon collimator was measured. A SEM image of the prepared sample was taken in order to observe the surface of the CsI:Tl filled in the silicon collimator. In addition, a cross-sectional image of the sample was taken by X-ray computed tomography (CT) using a photon-counting X-ray line sensor (XC-HYDRA FX20, X-Counter Inc.) to investigate the filling state of CsI:Tl in the silicon collimator.

The scintillation light in the silicon collimator was observed. Images of the sample irradiated with X-rays were taken with a digital single-lens reflex camera with a CMOS sensor (D5300, Nikon). A schematic diagram of the experiment is shown in Fig. 3. A profile of each pixel value was calculated along the red line in Fig. 5. Furthermore, the values for each CMOS pixel were summed to create a profile because the values for each collimator pixel are the integrated output when a silicon collimator is used in a detector. The uniformity of emitted light was evaluated by comparing these two profiles.

\subsection{Characteristics of luminescence of CsI:TI for silicon collimator}

The characteristics of the luminescence of CsI:Tl are affected by the concentration of $\mathrm{Tl}$ in CsI used as an activator. Different samples of CsI:Tl were prepared with different heating durations, and a silicon photodiode was used to detect optical photons generated by the scintillator in the silicon collimator. Since the quantum efficiency of this photodiode was calculated from 400 to $600 \mathrm{~nm},{ }^{(13)}$ the integrated emission intensity in this region of interest (ROI) was measured to attain optimum filling conditions. The emission characteristic spectrum of CsI:Tl for each heating time during X-ray irradiation (HSY-1021, HITACHI) was 


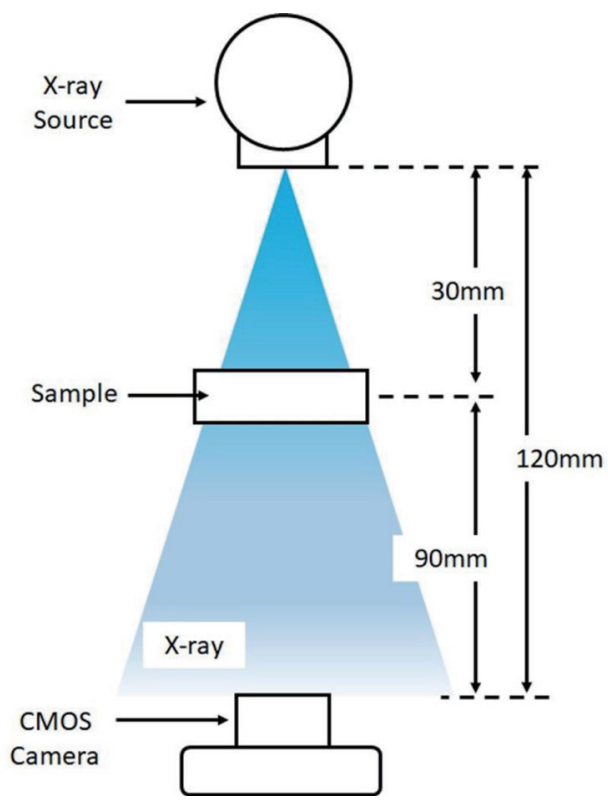

Fig. 3. (Color online) Schematic diagram of observing scintillation light in silicon collimator.

measured with a spectrometer (Ocean Optics USB4000). A tube voltage of $150 \mathrm{kV}$ with a current of $500 \mu \mathrm{A}$ was used to produce X-rays. The measured spectrum was integrated in the range of 500 to $600 \mathrm{~nm}$ to determine the integrated emission intensity for the photodiode. The $\mathrm{Tl}$ concentration of these filled samples was measured by inductivity coupled plasma-atomic emission spectroscopy (ICP-AES) (Optima 2100DV, PerkinElmer Inc.), and these results were taken as the indices of the relationship between the luminescence characteristics and the heating duration.

\subsection{Spatial resolution of silicon collimator}

The Modulation Transfer Function (MTF) curves in terms of the spatial resolution of the fabricated samples were measured from edge images by using a tungsten edge phantom method for each scintillator with a different $\mathrm{Tl}$ concentration. ${ }^{(13,14)}$ A CMOS sensor was used to take the edge image of the tungsten plate attached to the samples. The edge images within the ROI were acquired in order to obtain edge spread function (ESF) data. The ESF was differentiated to obtain the line spread function (LSF), and the MTF was calculated from the Fourier transform of the LSF data, where the value at zero frequency was normalized to one to obtain the $M T F^{(15)}$ The edge method is a LSF transformation method used to derive the LSF, $l(x)$, as the first derivative $e(x)$ of the ESF as follows. ${ }^{(16)}$

$$
l(x)=\frac{d(e(x))}{d(x)}
$$


The $M T F$ at spatial frequency $f$ is calculated as

$$
\operatorname{MTF}(f)=\left|\int_{-\infty}^{+\infty} l(x) \exp (-i 2 \pi f x) d x\right| .
$$

The edge images were obtained from two scintillator samples of (A) a commercial CsI:T1 plate (GPXS J13113, HAMAMATSU) and (B) CsI:Tl filled in a silicon collimator. Sample (B) was fabricated under the optimum filling conditions.

\section{Results and Discussion}

\subsection{Filling condition of CsI:Tl in silicon collimator}

The silicon collimator was filled with the CsI:Tl scintillator by the melting process. A SEM image of the surface of the prepared sample is shown in Fig. 4(a). A cross-sectional image obtained by X-ray CT to confirm the filling state of CsI:Tl in the silicon collimator is shown in Figs. 4(b) and 4(c). It can be observed that the holes of the silicon collimator are finely packed with CsI:Tl. Since the attenuation coefficient of CsI:Tl is larger than that of silicon, the X-ray CT image shows CsI:Tl in white. Several white squares in the shape of silicon collimator holes were observed. In Figs. 4(b) and 4(c), the edge of the collimator is blurred with ring artifacts due to CT imaging, but it was confirmed that the holes of the silicon collimator were completely filled with CsI:Tl from top to bottom.

The luminescence of the sample during X-ray irradiation and the profile along the red line in Fig. 5(a) were compared to observe the scintillation light of the CsI:Tl filled in the silicon collimator. In the profile, the sharp decrease in the gray value every $100 \mu \mathrm{m}$ indicates the optical separation effect of the silicon collimator. The result of integration of the gray value for each pixel of the silicon collimator is shown in Fig. 5(b). The integrated maximum value was

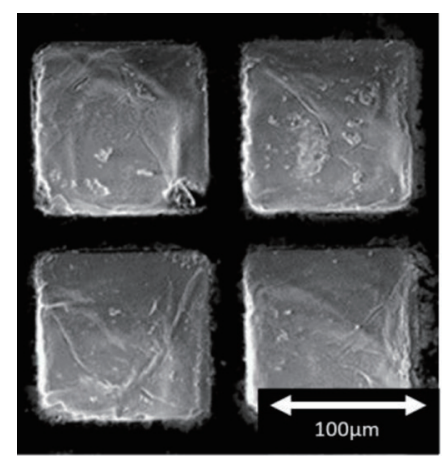

(a)

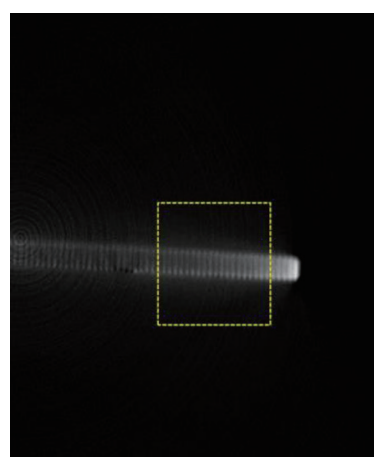

(b)

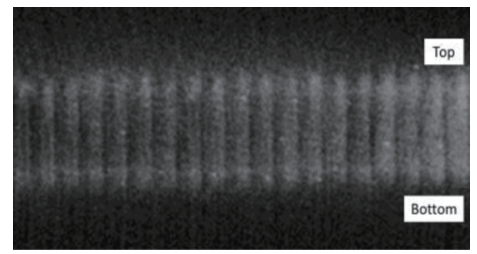

(c)

Fig. 4. (Color online) (a) SEM image of CsI:Tl in silicon collimator. (b) Partial image of X-ray tomography of silicon collimator. (c) Enlarged view of the area surrounded by yellow lines. 


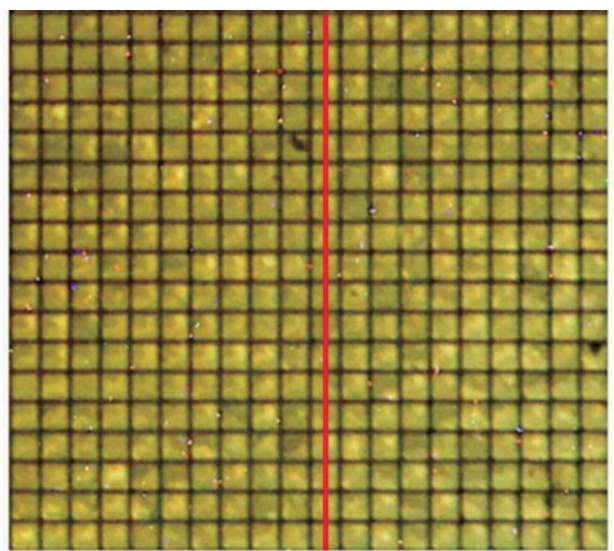

(a)

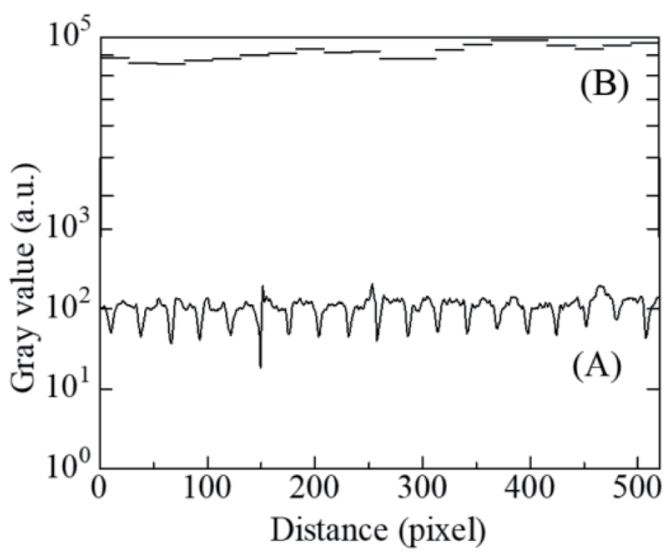

(b)

Fig. 5. (Color online) (a) Image of scintillation light in silicon collimator with CMOS sensor. (b) (A) Profiling along red line in (a) and (B) integrating each pixel along red line in (a).

observed to be $9.8 \times 10^{4}$ and the minimum value was $8.5 \times 10^{4}$. The variation in the values was about $13 \%$. From these results, it was confirmed that the silicon collimator was sufficiently filled with CsI:Tl, whose melting parameters are considered suitable for use in the silicon collimator, although there was some variability.

\subsection{Luminescence characteristics of CsI:Tl in silicon collimator}

Samples of CsI:Tl with different $\mathrm{Tl}$ concentrations were prepared with different heating times to determine the dependence of luminescence on the $\mathrm{Tl}$ concentration. The emission characteristics of these samples and the concentration of $\mathrm{Tl}$ were measured and analyzed. The characteristic spectrum obtained for each concentration is shown in Fig. 6(a). The integrated emission intensity obtained by integrating over the ROI of the photodiode (left axis) and the peak wavelength (right axis) are shown in Fig. 6(b). Both the integrated emission intensity and the peak emission wavelength increase in direct proportion to the Tl concentration. CsI:Tl with a $\mathrm{Tl}$ concentration of $0.69 \mathrm{~mol} \%$ prepared with 2 min melting has the highest integrated emission intensity, and the peak wavelength of this sample is in the ROI of the Si photodiode. Therefore, filling a silicon collimator with CsI:Tl using the melting process for 2 min results in a detector suitable for mounting.

\subsection{Spatial resolution of silicon collimator}

The effect of the silicon collimator on the spatial resolution of scintillator-type X-ray detector was measured and verified for various samples by using the tungsten edge phantom method. The edge images obtained from two fabricated scintillators are shown in Fig. 7 as insets (a) and (b). Figure 7 shows the MTF curves obtained by calculation from the edge images of samples (A) and (B). When the spatial frequency was $5.0 \mathrm{lp} / \mathrm{mm}$, the $M T F$ values of the samples were 


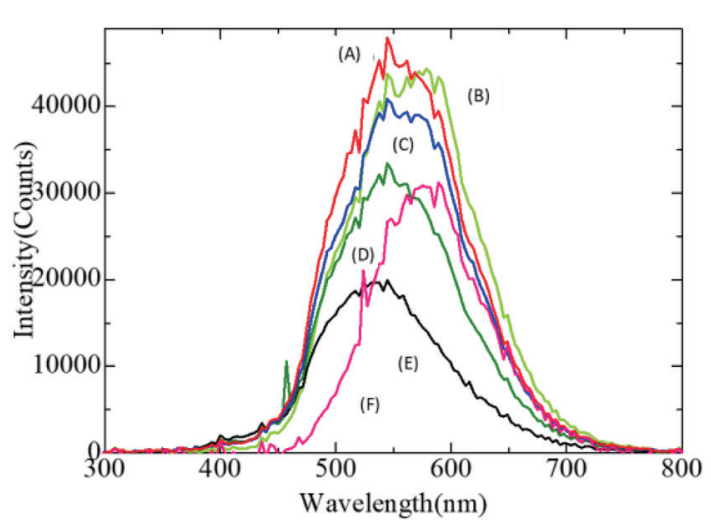

(a)

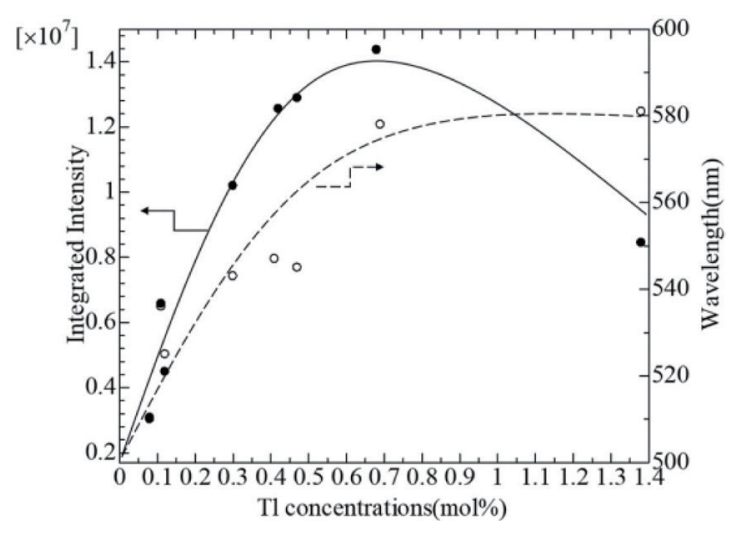

(b)

Fig. 6. (Color online) (a) Characteristics of luminescence of samples with $\mathrm{Tl}$ concentrations of (A) $0.69 \mathrm{~mol} \%$, (B) $0.47 \mathrm{~mol} \%$, (C) $0.41 \mathrm{~mol} \%$, (D) $0.30 \mathrm{~mol} \%$, (E) $0.11 \mathrm{~mol} \%$, and (F) $1.38 \mathrm{~mol} \%$. (b) Integrated emission intensity obtained by integrating the ROI of the photodiode (left scale) and peak wavelength (right scale).

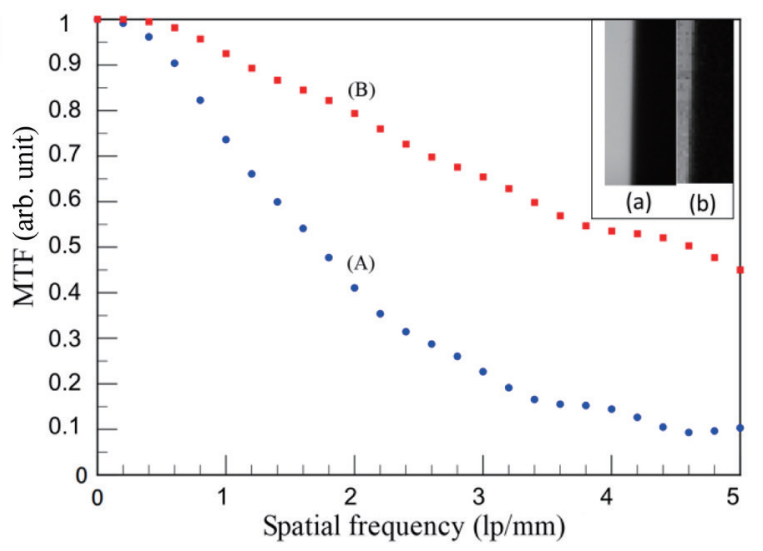

Fig. 7. (Color online) Image of tungsten edge taken by (a) commercial CsI:Tl plate and (b) fabricated CsI:Tl filled in silicon collimator. Curves (A) and (B) show the MTF values calculated from the edge images (a) and (b).

0.10 and 0.42 , respectively. This improvement in the spatial resolution can be attributed to the fact that the silicon collimator optically separates CsI:T1 and suppresses the diffusion of light emission. The $M T F$ values at a spatial frequency of $5.0 \mathrm{lp} / \mathrm{mm}$ reported in other papers on improving the spatial resolution of scintillator detectors with CsI:Tl columnar crystals are about 0.14 by Fedorov et al. in $2006,{ }^{(4)} 0.15$ by Kim et al. in $2018,{ }^{(15)}$ and 0.18 by Miller et al. in $2005 .{ }^{(8)}$ The MTF value of 0.42 in this paper is higher than the previously reported values. When CsI is separated by columnar crystals and light having an angle that does not satisfy the total reflection condition is incident on the boundary, the light is transmitted to the adjacent columnar crystal. ${ }^{(17)}$ The light does not affect adjacent pixels in the collimator because the silicon does not permit the transmission of visible light. The absorption of visible light by silicon does not affect the MTF because the MTF does not depend on the light intensity. Our results confirm that the silicon collimator improves the spatial resolution of a scintillator-type indirect X-ray imaging detector because the silicon collimator completely separates CsI:Tl crystals. 


\section{Conclusion}

A pixel-structured scintillator with a silicon collimator was successfully fabricated and showed high-spatial-resolution imaging performance in an indirect X-ray imaging detector. The pixel-structured silicon collimator was filled with CsI:Tl as a scintillator material after optimizing the filling conditions. The spatial resolution of the silicon collimator with CsI:TI was evaluated and its $M T F$ value was higher than that of the scintillator without the collimator and columnar crystals of CsI:Tl. As a result, the silicon collimator is promising for improving the spatial resolution of the scintillator for indirect-type X-ray imaging detectors.

\section{Acknowledgments}

This research was supported by the Cooperative Research Project of the Research Center for Biomedical Engineering, Research Institute of Electronics, Shizuoka University. We appreciate the personal communication with Nicholas Eib, Madhu Vora, Anand Sheel, and Brian Rodricks of Terapede Systems Inc., USA.

\section{References}

1 B. Kim, B. K. Cha, H. Jeon, Y. K. Chi, and G. Cho: Nucl. Instrum. Methods 579 (2007) 205. https://doi. org/10.1016/j.nima.2007.04.040

2 B. K. Cha, D. H. Lee, B. Kim, C. H. Seo, S. Jeon, and Y. Huh: J. Korean Phys. Soc. 59 (2011) 3670. https://doi. org/10.3938/jkps.59.3670

3 G. Lubberts: J. Opt. Soc. Am. 58 (1968) 1475. https://doi.org/10.1364/JOSA.58.001475

4 A. Fedorov, A. Lebedinsky, and O. Zelenskaya: Nucl. Instrum. Methods 564 (2006) 328. https://doi. org/10.1016/j.nima.2006.04.037

5 Y. H. Lei, X. Liu, J. C. Guo, and Z. G. Zhao: Chin. Phys. B 20 (2011) 042901. https://doi.org/10.1088/16741056/20/4/042901

6 W. Zhao, G. Ristic, and J. A. Rowlands: Med. Phys. 31 (2004) 2594. https://doi.org/10.1118/1.1782676

7 A. Ananenko, A. Fedorov, A. Lebedinsky, P. Mateychenko, V. Tarasov, and Y. Vidaj: Semicond. Phys. Quantum Electron. Optoelectron. 7 (2004) 297. https://doi.org/10.15407/spqeo7.03.297

8 S. Miller, V. Gaysinskiy, I. Shestakova, and V. V. Nagarkar: Penetrating Radiation Systems and Applications VII. International Society for Optics and Photonics 5923 (2005) 59230F. https://doi.org/10.1117/12.625927

9 R. Mao, L. Zhang, and R. Y. Zhu: IEEE Trans. Nucl. Sci. 55 (2008) 2425. https://doi.org/10.1109/ TNS.2008.2000776

10 H. W. Lau and G. J. Parker: Appl. Phys. Lett. 67 (1995) 1877. https://doi.org/10.1063/1.114362

11 T. Sato, K. Niita, N. Matsuda, S. Hashimoto, Y. Iwamoto, S. Noda, T. Ogawa, H. Iwase, H. Nakashima, T. Fukahori, K. Okumura, T. Kai, S. Chiba, T. Furuta, and L. Sihver: J. Nucl. Sci. Technol. 50 (2013) 913.

12 Y Liu, C. P Chen, H. B Li, C. H Tang, C. Y Chang, L. Hou, W. P. Lai, J. Li, S. T. Lin, C. S. Luo, J. F. Qiu, H. Y Sheng, C. C. Wang, M. Z. Wang, S. C. Wang, H. T. Wong, B. Xin, Q. Yue, D. X. Zhao, S. Q. Zhao, Z. Y. Zhou, and B. A. Zhuang: Nucl. Instrum. Methods 482 (2001) 125. https://doi.org/10.1016/S0168-9002(01)01458-9

13 V. Saveliev and V. Golovin: Nucl. Instrum. Methods 442 (2000) 223. https://doi.org/10.1016/S01689002(99)01225-5

14 IEC 62220-1-2. Medical Electrical Equipment-Characteristics of Digital X-ray Imaging Devices Part 1-2: Deteration of Detective Quantum Efficiency_Detectors Used in Dynamic Imaging, International Electrotechnical Commission (Switzer Land Geneva, 2008).

15 M. S. Kim, K. T. Lim, G. Kim, and G. Cho: Nucl. Instrum. Methods 882 (2018) 84. https://doi.org/10.1016/ j.nima.2017.11.023

16 D. Williams and P. D. Burns: Image Quallty and System Performance 5294 (2004) 93. https://doi. org $/ 10.1117 / 12.532405$

17 D. M. Schlosser, R. Hartmann, D. Kalok, A. Bechteler, A. Abboud, M. Shokr, T. Conka, U. Pietsch, and L. Struder: J. Instrum. 12 (2017) P04009. https://doi.org/10.1088/1748-0221/12/04/P04009 
